\title{
Terahertz Pulse Imaging of Micro-metastatic Lymph Nodes in Early-stage Cervical Cancer Patients
}

\author{
Euna Jung, Meehyun Lim, Kiwon Moon, Youngwoong Do, Soonsung Lee, and Haewook Han* \\ Department of Electrical and Computer Engineering, POSTECH, Pohang 790-784, Korea \\ Hyuck Jae Choi and Kyoung-Sik Cho \\ Department of Radiology and Research Institute of Radiology, Asan Medical Center, \\ University of Ulsan Collage of Medicine, Seoul 138-736, Korea \\ Kyu-Rae Kim \\ Department of Pathology, Asan Medical Center, University of Ulsan Collage of Medicine, Seoul, 138-736, Korea
}

(Received March 2, 2011 : revised March 18, 2011 : accepted March 18, 2011)

\begin{abstract}
Lymph node metastasis is an important prognostic factor in cervical cancer patients. We report $\mathrm{THz}$ imaging for detecting micro-metastatic foci in the lymph nodes of early-stage uterine cervical cancer patients. Five paraffin-embedded metastatic lymph nodes from two cervical cancer patients were imaged using a THz time-domain spectroscopy system in the reflection mode. The size and shape of the tumor regions were compared with those from histopathologic examinations. The metastatic portions of lymph nodes as small as $3 \mathrm{~mm}$ were well delineated by $\mathrm{THz}$ imaging. The reflected peak amplitudes were lower in metastatic portions than in the normal portions of lymph nodes, and the difference in their peak-to-peak amplitudes was $\sim 5 \%$.
\end{abstract}

Keywords: Terahertz imaging, Cervical cancer, Lymph node metastasis

OCIS codes : (110.6795) Terahertz imaging; (170.3880) Medical and biological imaging; (300.6495) Spectroscopy, terahertz

\section{INTRODUCTION}

Cervical cancer is the second most common malignancy in women worldwide and is the only major gynecological malignancy clinically staged according to the International Federation of Obstetrics and Gynecology (FIGO) recommendations [1]. The prognostic factors identified in cervical cancer patients are tumor size, depth of stromal invasion, presence of lymphovascular tumor emboli, histologic type, and invasion of surgical margins [2, 3]. Lymph node metastasis is also closely associated with poor prognosis and decreased survival rate [4]; moreover, this metastasis is an indicator for adjuvant radiotherapy [5].

Computed tomography (CT), magnetic resonance imaging (MRI), and positron emission tomography-CT (PET-CT) have been used to detect metastatic lymph nodes in cervical cancer patients, but their accuracies were lower than expected $[6,7]$. In one study of cervical cancer patients negative for metastatic lymph nodes by MRI, the sensitivity of PET in detecting metastatic lymph nodes was only $10 \%$ [8]. This low diagnostic performance was attributed to the adoption of inaccurate size criteria and a possible substantial number of small metastatic lymph nodes. Although surgical lymph node staging is an option, it is not routinely recommended because it is a specialized procedure that increases the time and cost of the surgery, and it leads to operation-related complications $[9,10]$. The accurate diagnosis of metastatic lymph nodes is of clinical importance, the lack of sensitive diagnostic methods indicates the need for additional imaging modalities.

In recent years, terahertz $(\mathrm{THz})$ imaging technology has shown great promise and has increasingly been performed

\footnotetext{
*Corresponding author: hhan@postech.ac.kr

Color versions of one or more of the figures in this paper are available online.
} 
for cancer diagnosis and treatment [11-13]. Although THz imaging may improve the diagnosis of metastatic lymph nodes in cervical cancer patients, it has not yet been evaluated in this setting. Therefore, we report a pilot study for $\mathrm{THz}$ imaging to detect lymph node micro-metastases in earlystage cervical cancer patients, which are usually undiagnosed by conventional imaging methods.

\section{MATERIALS AND METHODS}

\subsection{Sample Preparation}

Two patients, aged 45 and 50 years, with FIGO stage IB1 and squamous cell carcinoma cervical cancer, respectively, underwent conventional lymphadenectomy combined with radical hysterectomy. Dissected lymph nodes were embedded in paraffin for histologic diagnosis and $\mathrm{THz}$ evaluation. Five paraffin-embedded metastatic lymph nodes from the two patients (three from one patient, two from the other) were sliced at 5-mm intervals in the longitudinal plane, and $4-\mu \mathrm{m}$ sections were stained with hematoxylin and eosin. Because of the retrospective design of this study, our institutional review boards did not require approval or informed consent for the review of the patients' specimens.

\subsection{Image Acquisition and Measurement}

All lymph node specimens were imaged using a $\mathrm{THz}$ time-domain spectroscopy (THz TDS) system operating in reflection mode [12]. The $\mathrm{THz}$ pulse was generated by an InAs wafer pumped by a Ti:Sapphire laser with a center wavelength of $790 \mathrm{~nm}$, a pulse width of $100 \mathrm{fs}$, and a repetition rate of $80 \mathrm{MHz}$. The THz pulse was focused on the specimen at a normal incidence by using an off-axis parabolic mirror, and the reflected $\mathrm{THz}$ pulse was detected by a photoconductive antenna fabricated on a low-temperature grown GaAs. The entire THz TDS system was enclosed in a chamber and continuously purged with dry air to eliminate $\mathrm{THz}$ absorption by water vapor in the air. The bandwidth of the imaging setup ranged from 0.2 to $2.0 \mathrm{THz}$ with a signalto-noise ratio of 2000:1. Two-dimensional THz images were obtained by raster-scanning of specimens in the $\mathrm{x}-\mathrm{y}$ plane. Each scan area was $\sim 20 \mathrm{~mm} \times 20 \mathrm{~mm}$, consisting of 40 $\times 40$ pixels with a pixel pitch of $0.5 \mathrm{~mm}$, and the image quality was enhanced by using a spline interpolation.

$\mathrm{THz}$ images were obtained using the normalized peak-topeak amplitude of reflected $\mathrm{THz}$ pulses at each pixel of the specimen, $E(x, y) / E_{0}$, where $E_{0}$ is the minimum peakto-peak amplitude of $\mathrm{THz}$ pulses within the specimen. After Fourier transformation of the measured $\mathrm{THz}$ pulses, the frequency dependent reflection coefficient at each interface between the specimen and air, $r(x, y, f)$, was calculated as

$$
r(x, y, f)=\frac{1-n_{p}(f)-\Delta n(x, y, f)}{1+n_{p}(f)+\Delta n(x, y, f)},
$$

where $f$ is frequency, $n_{p}(f)$ is the refractive index of paraffin, and $\Delta n(x, y, f)=n(x, y, f)-n_{p}(f)$ is the difference in refractive index between the lymph node and paraffin. Equation (1) can be rearranged as

$$
\frac{\Delta n(x, y, f)}{n_{p}(f)}=\frac{1}{n_{p}(f)} \frac{[1-\gamma(x, y, f)]\left[1-n_{p}^{2}(f)\right]}{\gamma(x, y, f)\left[1-n_{p}(f)\right]+\left[1+n_{p}(f)\right]},
$$

where $\gamma(x, y, f)$ is the reflection coefficient of the lymph node, normalized by the reflection coefficient of paraffin $r_{p}(f)$,

$$
\gamma(x, y, f)=\frac{r(x, y, f)}{r_{p}(f)} .
$$

Because the frequency dependence of $n_{p}(f)$ is negligible in the THz frequency range used in experiments [14], $n_{p}(f)$ is 1.5 and $\chi(x, y, f) \approx E(x, y) / E_{0}$. THz images were obtained by mapping the relative refractive index using equation (2) and evaluated by comparing the size and shape of the region of contrast with the size and shape of the tumor region identified by histopathologic examination of the corresponding stained lymph node slices under an optical microscope.

\section{EXPERIMENTAL RESULTS AND DISCUSSION}

Figure 1 shows reflected $\mathrm{THz}$ pulses and spectral amplitudes of lymph node specimens at the selected pixels indicated in Fig. 2. For all specimens, the reflected peak amplitudes were smaller for the metastatic than for the non-metastatic portions of lymph nodes, as was the spectral amplitude in the $\mathrm{THz}$ frequency range. These reductions in peak amplitudes in the metastatic region were primarily due to changes in the refractive index, as shown in Fig. 2.

The $\mathrm{THz}$ images clearly differentiated metastatic regions from non-metastatic regions, even for 3-mm metastatic foci (Fig. 2) and showed good correlations with histopathologic findings. This differentiation, however, was not evident in the optical images. THz images were generated on a color scale by mapping two image parameters: $E(x, y) / E_{0}$ and $\Delta n(x, y, f) / n_{p}$. The metastatic foci ranged in size from 3 to $12 \mathrm{~mm}$, with a mean size of $6 \pm 0.5 \mathrm{~mm}$. There were no significant differences in contrast between two $\mathrm{THz}$ images by using peak-to-peak amplitudes and refractive index changes, demonstrating that $\mathrm{THz}$ images can also determine the optical properties, such as refractive index, of lymph node specimens. We observed that the refractive index decreased in regions of metastatic lymph nodes. The mean refractive indices of the non-metastatic and metastatic portions of lymph nodes were $1.84 \pm 0.04$ and $1.76 \pm 0.03$, respectively. The index difference between non-metastatic and metastatic portions 
(a1)
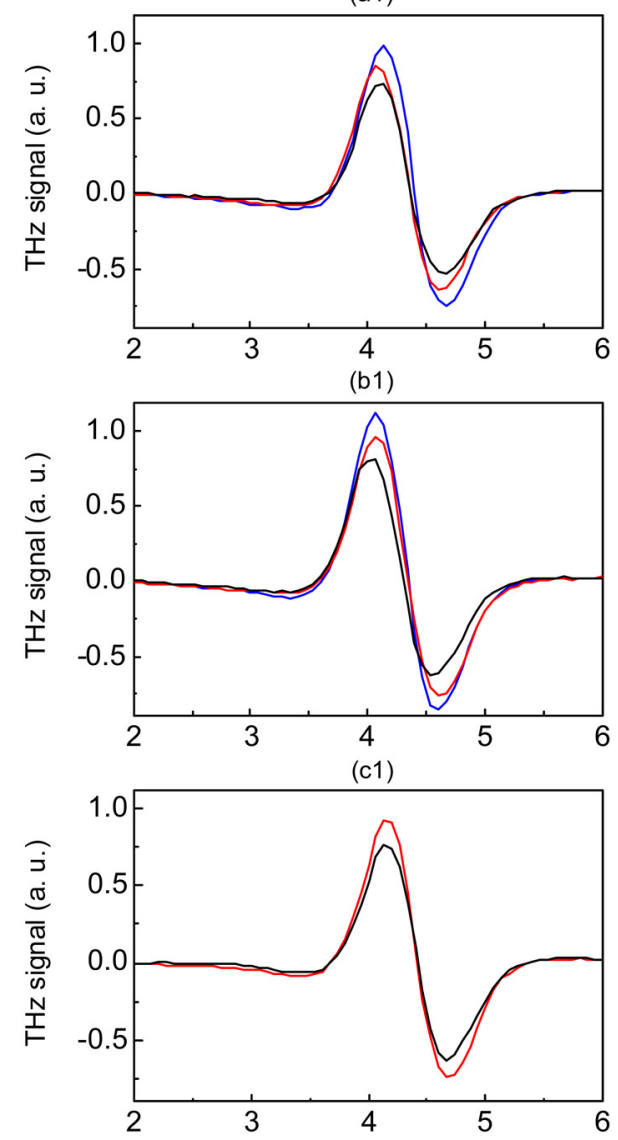

(d1)

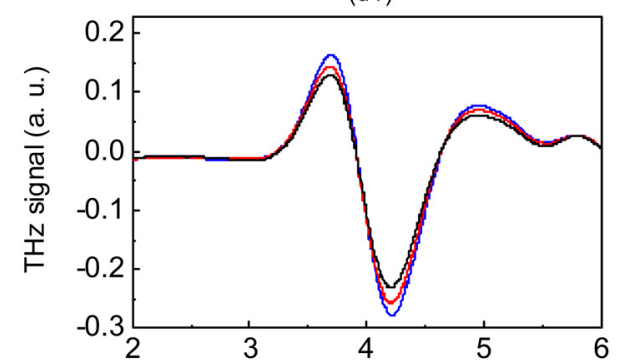

(e1)

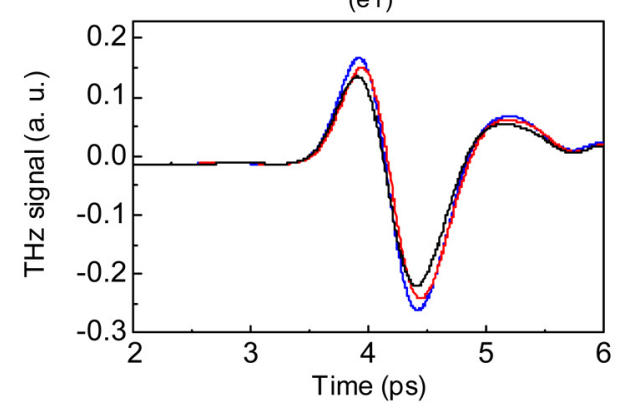

(a2)
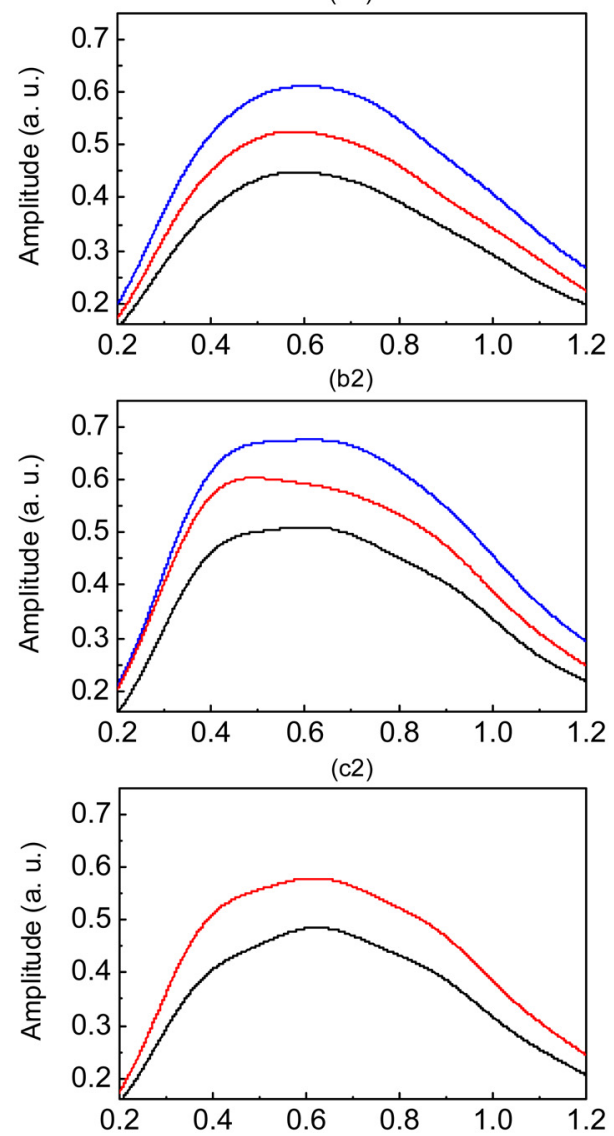

(d2)
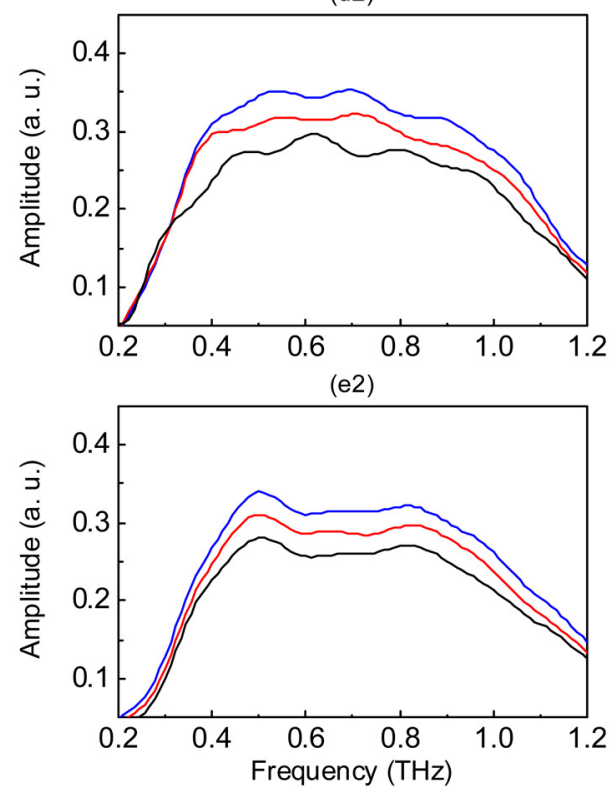

FIG. 1. Reflected THz pulses (left) and THz spectral amplitudes (right) of paraffin (black), non-metastatic (blue), and metastatic (red) portions of five lymph nodes at the selected pixels indicated in Fig. 2.

of lymph nodes was $4.7 \pm 0.5 \%$.

Lymph node metastasis is an important factor influencing prognosis and therapeutic strategy in patients with cervical cancer. Although CT and MRI have been used to detect metastatic lymph nodes, including measurements of size, shape, presence of necrosis, and enhancement characteristics, these methods cannot efficiently differentiate malignant from hyperplastic lymph nodes [15-17]. Many of these metastatic 
(a1)

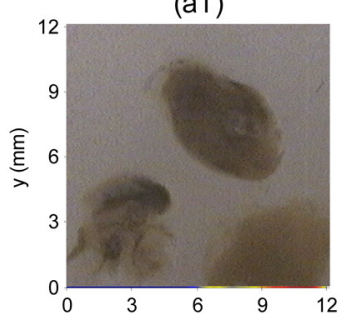

(b1)

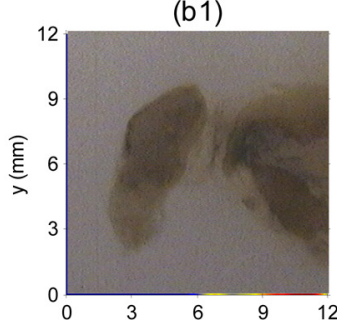

(c1)

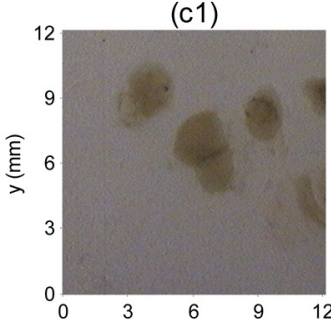

(d1)

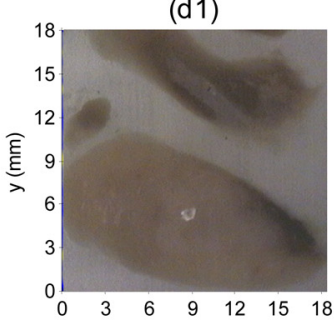

(e1)

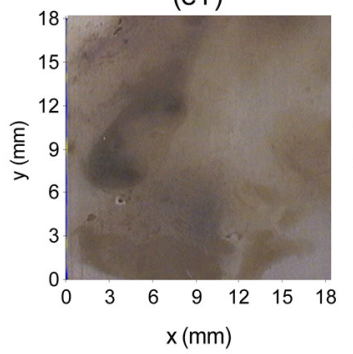

(a2)

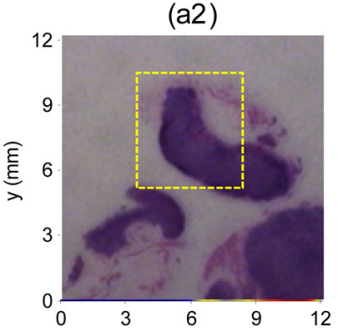

(b2)

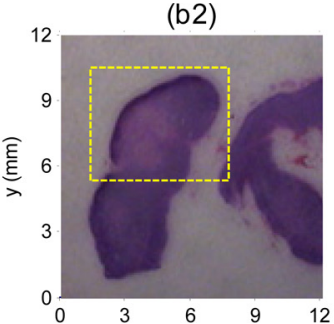

(c2)

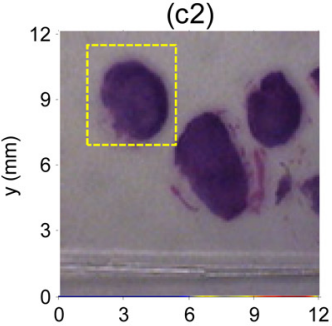

(d2)

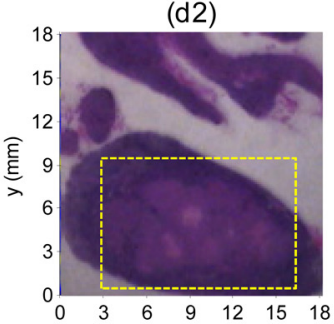

(e2)

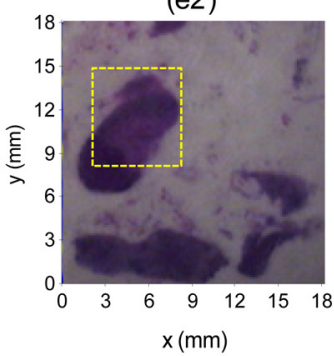

(a3)

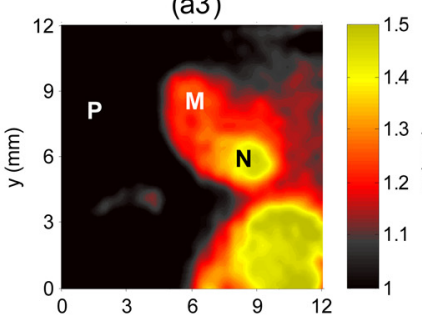

(b3)

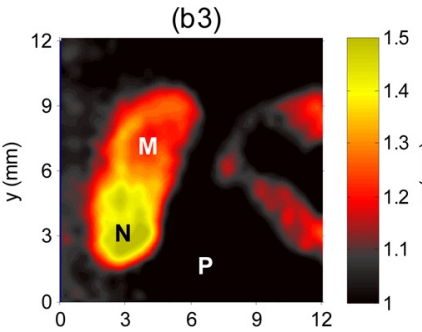

(c3)

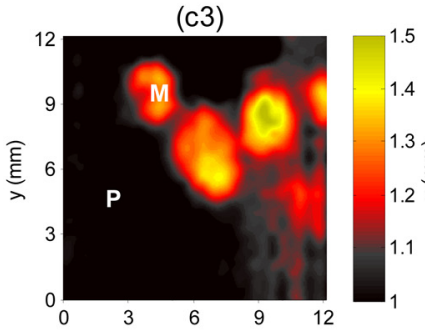

(d3)

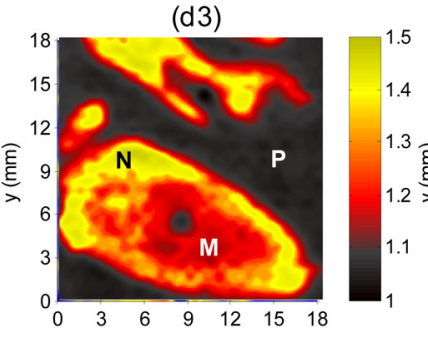

(e3)

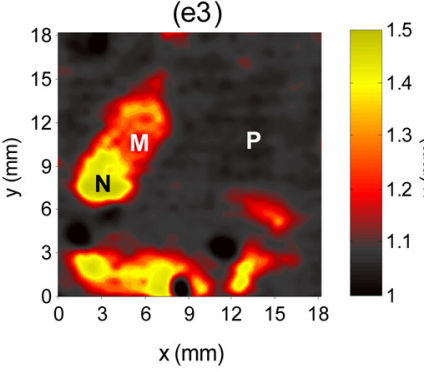

(a4)

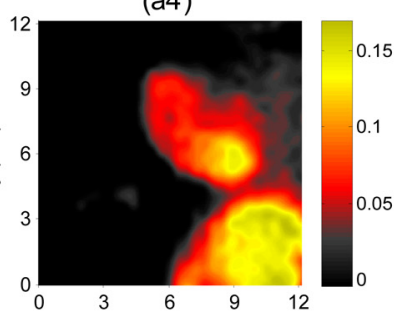

(b4)

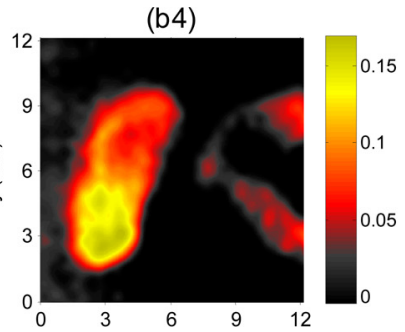

(c4)

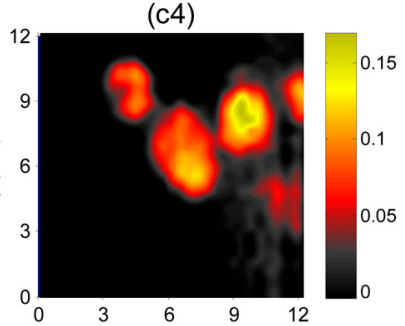

(d4)

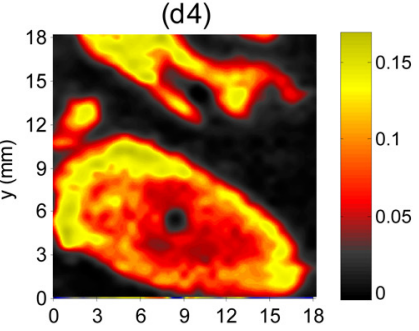

(e4)

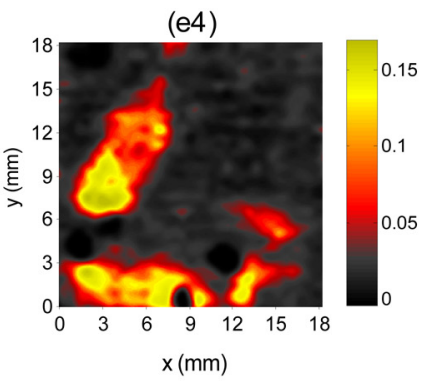

FIG. 2. Optical (column 1), histopathologic (column 2), and THz images (columns 3 and 4) of five paraffin-embedded metastatic lymph nodes from two patients. The THz images were obtained using $\mathrm{E}(\mathrm{x}, \mathrm{y}) / \mathrm{E}_{0}$ (column 3$)$ and $\Delta \mathrm{n}(\mathrm{x}, \mathrm{y}, \mathrm{f}) / \mathrm{n}_{\mathrm{p}}(\mathrm{column} 4)$. Selected pixels are indicated in column 3 (P: paraffin, M: metastatic portion, $\mathrm{N}$ : non-metastatic portion). The dashed boxes indicate the metastatic portion identified by histopathologic examination. The THz images showed good correlations with histopathologic results.

lymph nodes are smaller than $1 \mathrm{~cm}$, the usual diagnostic size criteria for metastatic lymph nodes, thereby reducing the diagnostic accuracy of CT and MRI [18]. Although PET scanning diagnoses metastatic lymph nodes by assessing the abnormal accumulation of FDG, PET is expensive and less widely available, and has lower spatial resolution. The pooled sensitivities of CT, MRI, and PET were found to be only $52 \%, 38 \%$, and $54 \%$, respectively, in region- or nodebased comparisons, which are too low to replace surgical staging $[19,20]$. Thus, there are as-of-yet unmet clinical needs for the accurate imaging diagnosis of metastatic lymph nodes and detection of small metastatic nodes, i.e., diagnoses that better reflect the actual histological processes.

Our pilot study showed that $\mathrm{THz}$ images delineated metastatic 
foci in lymph nodes to an extent similar to those of histopathologic methods. Although the overall correlation of metastatic foci and $\mathrm{THz}$ images were good, peak reflection amplitudes at the edges of the lymph nodes were lower because of averaging with the adjacent paraffin, which has much lower reflected peak amplitudes. This edge effect may be responsible for the false positive findings in benign portions of the lymph nodes. We found that the smallest metastatic focus delineated on $\mathrm{THz}$ imaging was approximately $3 \mathrm{~mm}$ in its largest dimension. Although these were specimen images, we believe that our findings are meaningful as a pilot study for future investigations of this imaging modality in the diagnosis of micro-metastatic lymph nodes, which are usually undiagnosed by conventional imaging methods.

Earlier studies have suggested that water content is a major contributory factor for contrast in $\mathrm{THz}$ images since tumors generally contain a higher water content than normal tissue and liquid water has high attenuation for $\mathrm{THz}$ waves [21-23]. Although several other studies have used paraffinembedded tissues [24-26], the fundamental mechanisms underlying the contrast in $\mathrm{THz}$ images of the dehydrated paraffin-embedded samples that differentiate tumor from normal tissue are not clear at this stage. However, the different contrasts for tumors and normal tissues in $\mathrm{THz}$ images of dehydrated paraffin-embedded samples may be due to other components, including cell density, proteins, and DNA. Thus, paraffin-embedded tissue samples may be useful for assessing cancer biology using $\mathrm{THz}$ imaging and for investigating $\mathrm{THz}$ contrast mechanisms, other than the effects of water.

\section{CONCLUSION}

We have shown here that $\mathrm{THz}$ imaging can clearly delineate the metastatic portions of the lymph nodes as small as 3 $\mathrm{mm}$. Two THz images by using peak-to-peak amplitudes and refractive index changes were almost identical, demonstrating that $\mathrm{THz}$ images can also determine refractive indices of lymph node specimens. THz imaging is a promising technique for the detection of micro-metastatic lymph nodes that cannot be detected by conventional imaging methods, but technical improvements are needed prior to its clinical application.

\section{ACKNOWLEDGMENT}

This research was supported by the Basic Science Research Program (2009-0083512), by the Priority Research Centers Program (2010-0029711) through the National Research Foundation of Korea (NRF) funded by the Ministry of Education, Science and Technology, and by the Brain Korea 21 Project in 2011.

\section{REFERENCES}

1. D. M. Parkin, F. Bray, J. Ferlay, and P. Pisani, "Estimating the world cancer burden: Globocan 2000," Int. J. Cancer 94, 153-156 (2001).

2. M. M. Juretzka, K. C. Jensen, T. A. Longacre, N. N. Teng, and A. Husain, "Detection of pelvic lymph node micrometastasis in stage IA2-IB2 cervical cancer by immunohistochemical analysis," Gynecol. Oncol. 93, 107-111 (2004).

3. P. Marchiole, A. Buenerd, M. Benchaib, K. Nezhat, D. Dargent, and P. Mathevet, "Clinical significance of lympho vascular space involvement and lymph node micrometastases in early-stage cervical cancer: a retrospective case-control surgico-pathological study," Gynecol. Oncol. 97, 727-732 (2005).

4. N. Takeshima, K. Yanoh, T. Tabata, K. Nagai, Y. Hirai, and K. Hasumi, "Assessment of the revised international federation of gynecology and obstetrics staging for early invasive squamous cervical cancer," Gynecol. Oncol. 74, 165-169 (1999).

5. Y. Tanaka, S. Sawada, and T. Murata, "Relationship between lymph node metastases and prognosis in patients irradiated postoperatively for carcinoma of the uterine cervix," Acta Radiol. Oncol. 23, 455-459 (1984).

6. H. J. Choi, J. W. Roh, S. S. Seo, S. Lee, J. Y. Kim, S. K. Kim, K. W. Kang, J. S. Lee, J. Y. Jeong, and S. Y. Park, "Comparison of the accuracy of magnetic resonance imaging and positron emission tomography/computed tomography in the presurgical detection of lymph node metastases in patients with uterine cervical carcinoma: a prospective study," Cancer 106, 914-922 (2006).

7. A. D. Williams, C. Cousins, W. P. Soutter, M. Mubashar, A. M. Peters, R. Dina, F. Fuchsel, G. A. McIndoe, and N. M. deSouza, "Detection of pelvic lymph node metastases in gynecologic malignancy: a comparison of CT, MR imaging, and positron emission tomography," AJR Am. J. Roentgenol. 177, 343-348 (2001).

8. H. H. Chou, T. C. Chang, T. C. Yen, K. K. Ng, S. Hsueh, S. Y. Ma, C. J. Chang, H. J. Huang, A. Chao, T. I. Wu, S. M. Jung, Y. C. Wu, C. T. Lin, K. G. Huang, and C. H. Lai, "Low value of [18F]-fluoro-2-deoxy-D-glucose positron emission tomography in primary staging of early-stage cervical cancer before radical hysterectomy," J. Clin. Oncol. 24, 123-128 (2006).

9. J. R. van Nagell Jr., J. W. Roddick Jr., and D. M. Lowin, "The staging of cervical cancer: inevitable discrepancies between clinical staging and pathologic findinges," Am. J. Obstet. Gynecol. 110, 973-978 (1971).

10. J. Scheidler, H. Hricak, K. K. Yu, L. Subak, and M. R. Segal, "Radiological evaluation of lymph node metastases in patients with cervical cancer. A meta-analysis," JAMA 278, 1096-1101 (1997).

11. P. C. Ashworth, E. Pickwell-MacPherson, E. Provenzano, S. E. Pinder, A. D. Purushotham, M. Pepper, and V. P. Wallace, "Terahertz pulsed spectroscopy of freshly excised human breast cancer," Opt Express 17, 12444-12454 (2009).

12. A. J. Fitzgerald, V. P. Wallace, M. Jimenez-Linan, L. Bobrow, R. J. Pye, A. D. Purushotham, and D. D. Arnone, "Terahertz pulsed imaging of human breast tumors," Radiology 239, 533-540 (2006). 
13. V. P. Wallace, A. J. Fitzgerald, S. Shankar, N. Flanagan, R. Pye, J. Cluff, and D. D. Arnone, "Terahertz pulsed imaging of basal cell carcinoma ex vivo and in vivo," Br. J. Dermatol. 151, 424-432 (2004).

14. T. Enatsu, H. Kitahara, K. Takano, and T. Nagashima, "Terahertz spectroscopic imaging of paraffin-embedded liver cancer samples," in Proc. Conference on Infrared, Millimeter and Terahertz Electronics (Cardiff, UK, Sept. 2007), pp. 557-558.

15. A. D. King, G. M. Tse, A. T. Ahuja, E. H. Yuen, A. C. Vlantis, E. W. To, and A. C. van Hasselt, "Necrosis in metastatic neck nodes: diagnostic accuracy of CT, MR imaging, and US," Radiology 230, 720-726 (2004).

16. M. W. van den Brekel, H. V. Stel, J. A. Castelijns, J. J. Nauta, I. van der Waal, J. Valk, C. J. Meyer, and G. B. Snow, "Cervical lymph node metastasis: assessment of radiologic criteria," Radiology 177, 379-384 (1990).

17. M. G. Mack, J. Rieger, M. Baghi, S. Bisdas, and T. J. Vogl, “Cervical lymph nodes," Eur. J. Radiol. 66, 493-500 (2008).

18. H. J. Choi, S. H. Kim, S. S. Seo, S. Kang, S. Lee, J. Y. Kim, Y. H. Kim, J. S. Lee, H. H. Chung, J. H. Lee, and S. Y. Park, "MRI for pretreatment lymph node staging in uterine cervical cancer," AJR Am. J. Roentgenol. 187, W538-543 (2006).

19. H. J. Choi, W. Ju, S. K. Myung, and Y. Kim, "Diagnostic performance of computer tomography, magnetic resonance imaging, and positron emission tomography or positron emission tomography/computer tomography for detection of metastatic lymph nodes in patients with cervical cancer: meta-analysis," Cancer Sci. 101, 1471-1479 (2010).

20. H. Hricak, C. Gatsonis, D. S. Chi, M. A. Amendola, K.
Brandt, L. H. Schwartz, S. Koelliker, E. S. Siegelman, J. J. Brown, R. B. McGhee, Jr., R. Iyer, K. M. Vitellas, B. Snyder, H. J. Long 3rd, J. V. Fiorica, and D. G. Mitchell, "Role of imaging in pretreatment evaluation of early invasive cervical cancer: results of the intergroup study American college of radiology imaging network 6651-gynecologic oncology group 183," J. Clin. Oncol. 23, 9329-9337 (2005).

21. M. Bruehlmeier, U. Roelcke, P. Blauenstein, J. Missimer, P. A. Schubiger, J. T. Locher, R. Pellikka, and S. M. Ametamey, "Measurement of the extracellular space in brain tumors using 76Br-bromide and PET," J. Nucl. Med. 44, 1210-1218 (2003).

22. J. H. Chen, H. E. Avram, L. E. Crooks, M. Arakawa, L. Kaufman, and A. C. Brito, "In vivo relaxation times and hydrogen density at $0.063-4.85 \mathrm{~T}$ in rats with implanted mammary adenocarcinomas," Radiology 184, $427-434$ (1992).

23. K. F. Ross and R. E. Gordon, "Water in malignant tissue, measured by cell refractometry and nuclear magnetic resonance," J. Microsc. 128, 7-21 (1982).

24. P. Knobloch, C. Schildknecht, T. Kleine-Ostmann, M. Koch, S. Hoffmann, M. Hofmann, E. Rehberg, M. Sperling, K. Donhuijsen, G. Hein, and K. Pierz, "Medical THz imaging: an investigation of histo-pathological samples," Phys. Med. Biol. 47, 3875-3884 (2002).

25. T. Loffler, K. Siebert, S. Czasch, T. Bauer, and H. G. Roskos, "Visualization and classification in biomedical terahertz pulsed imaging," Phys. Med. Biol. 47, 3847-3852 (2002).

26. E. Berry, J. W. Handley, A. J. Fitzgerald, W. J. Merchant, R. D. Boyle, N. N. Zinov'ev, R. E. Miles, J. M. Chamberlain, and M. A. Smith, "Multispectral classification techniques for terahertz pulsed imaging: an example in histopathology," Med. Eng. Phys. 26, 423-430 (2004). 